\title{
Relación entre la estabilidad del core y el equilibrio postural en las habilidades biomotoras de los jóvenes atletas de baloncesto: una revisión sistemática Relationship between Core Stability and Postural Balance on the Biomotor Capabilities of Young Basketball Athletes: a systematic review. \\ Caio Bueno da Silva Sartori, Paulo Cesar Montagner, João Paulo Borin \\ Universidade Estadual de Campinas (Brasil)
}

Resumen. El baloncesto requiere exigencias físicas variadas, caracterizadas por esfuerzos intermitentes y situaciones de desequilibrio. Core Stability (EC) y Postural Balance (EP) son temas asociados como un factor significativo en la prevención de lesiones y dolor. Sin embargo, los resultados de los estudios en relación con las habilidades biomotoras son contrastantes, lo que indica que no hay efectos sobre el rendimiento deportivo en el contexto de alto rendimiento, pero es necesario profundizar las investigaciones sobre esta relación en la población adolescente.El objetivo del estudio fue verificar la relación entre la EC y la EP sobre las habilidades biomotoras en practicantes de baloncesto adolescentes. Utilizando la estrategia PICO, se realizaron búsquedas en las bases de datos PubMed y Scielo, buscando estudios experimentales con intervención de ejercicios EC / EP y pruebas de rendimiento atlético. Los resultados apuntan pocos estudios con efectos positivos sobre el rendimiento deportivo de los jóvenes atletas de baloncesto, pero no fue posible identificar pruebas sólidas de la influencia positiva del entrenamiento de EC / EP en las habilidades biomotoras de los adolescentes practicantes de baloncesto. Es necesario realizar más investigaciones sobre la influencia en los indicadores de rendimiento deportivo.

Palabras clave: Baloncesto, Adolescentes, Equilibrio Postural, Estabilidad Del Core y Rendimiento Atlético.

Abstract. Basketball demands varied physical demands, characterized by intermittent efforts and situations of imbalance. Core Stability (EC) and Postural Balance (EP) are themes that have been associated as a significant factor in the prevention of injuries and pain. However, the results of studies in relation to biomotor skills are contrasting, indicating that there are no effects on athletic performance in the context of high performance, but investigations on this relationship in the adolescent population need to be further investigated. The aim of the study was to verify the relationship between EC and PE on biomotor skills in adolescent basketball players. Using the PICOS strategy, searches were performed in the PubMed and Scielo databases, looking for experimental studies with EC / EP exercise intervention and athletic performance test. The results point to studies with positive effects on the athletic performance of young basketball athletes, however few among them used a specific additional intervention. There is a need for further investigation regarding the influence on athletic performance indicators.

Keywords: Basketball, Adolescents, Postural balance, Core stability and Athletic performance.

\section{Introducción}

El baloncesto requiere de exigencias físicas variadas, combinando capacidad aeróbica (Narazaki y col., 2009) y acciones de alta intensidad con un carácter anaeróbico (saltos, sprints, etc.) durante todo el partido (Mcinnes et al., 1995; Albaladejo et al., 2019). En este contexto, es necesario fuerza y equilibrio para soportar los contactos y golpes que conlleva el juego y realizar acciones deportivas con precisión (Shelling \& Torres-Ronda, 2013), así como reducir la incidencia de lesiones (Junior \& Gomes, 2014). Gollhofer (2003) cita el control postural y neuromuscular como contribuyen-

Fecha recepción: 10-06-21. Fecha de aceptación: 17-12-21

Caio Bueno da Silva Sartori Caio Sartori

caiobuenosartori@gmail.com tes clave para mejorar el rendimiento atlético en diferentes actividades, especialmente en condiciones perturbadas, donde la mayor sensibilidad de las vías aferentes parece reflejar una activación muscular más rápida y eficiente al comienzo del desarrollo de fuerza.

La estabilidad del core (EC) y el equilibrio postural (EP) son temas que han ido ganando terreno en las investigaciones científicas en las últimas décadas y ambos se han asociado como un factor importante en la prevención de lesiones y dolor (Richardson et al., 2002; Plisky et al., 2006). La EC puede interpretarse como la capacidad de controlar la posición y el movimiento del tronco sobre la pelvis y las extremidades inferiores para permitir una mejor producción, transferencia y control de la fuerza a los segmentos distales en actividades integradas de la cadena cinética (Kibler y col., 2006). EP se puede dividir en estático y dinámico. El equili- 
brio estático es la capacidad de mantener una base de apoyo con un movimiento mínimo. Dinámico es el mantenimiento o recuperación de una posición estable al realizar una tarea o sobre una superficie inestable (Hrysomallis, 2011).

Las formas de entrenar el core van desde ejercicios aislados (tabla isométrica, extensión lumbar, flexión de tronco, etc.) hasta los integrados y más dinámicos orientados a la acción deportiva, existen diferentes recomendaciones de ejercicios para deportistas, rehabilitación y población en general para entrenar el core (Behm et al, 2010). El entrenamiento del equilibrio está marcado por la presencia de inestabilidad, ya sea con el uso de dispositivos desestabilizadores (disco de equilibrio, pelota suiza, BOSU, etc.) o con desestabilización constante (ejercicios de resistencia, lanzamiento o recepción de pelota, uso de bandas elásticas, etc.), o con ambos (ZECH et al., 2010). Willardson (2007) afirma que el entrenamiento del equilibrio se puede considerar como entrenamiento del core, ya que las alteraciones afectan el centro de gravedad y deben ser suprimidas activando esta musculatura para hacer ajustes posturales y generar estabilidad.

Recientemente, se ha investigado la relación con as capacidades biomotoraspara verificar si el entrenamiento específico de estas habilidades puede influir positivamente en el rendimiento atlético (Zech et al., 2010). Este tipo de entrenamiento se utiliza en programas de entrenamiento atlético, utilizando equipos que generan inestabilidad, con la creencia de que mejorar EC / EP también mejora el rendimiento en el campo o en la cancha. Sin embargo, los resultados de estudios en esta dirección son contrastantes y no permiten llegar a un consenso (Sharrock et al., 2011). Bompa (2006) define las capacidades biomotoras como componentes físicos desarrollados por el cuerpo. La combinación de fuerza, velocidad, resistencia, coordinación, flexibilidad y equilibrio es fundamental para el éxito en las actividades motoras y vincular estas seis acciones es el objetivo del entrenamiento físico.

Después de revisar el entrenamiento central en el contexto de los atletas de alto rendimiento e identificar poca evidencia al respecto, Wirth y col. (2017) recomiendan el entrenamiento de fuerza tradicional para mejorar el rendimiento atlético. Okada y col. (2011) sugieren que el entrenamiento isométrico básico proporciona poco o ningún beneficio al rendimiento dinámico.

Por otro lado, Hoshikawa et al. (2013) encontraron una mejora en la fuerza de extensión de la cadera y el rendimiento del salto vertical con un entrenamiento de estabilidad complementario en atletas de fútbol jóvenes (12-13 años). Además de Distefano et al. (2013) y Shinkle et al. (2012) también identificaron relaciones significativas en atletas jóvenes.

Las investigaciones sobre la relación entre la EC / EF y el rendimiento deportivo en la población adolescente necesitan más cuerpo (Prieske et al, 2016), considerando los factores presentados y el vínculo entre equilibrio y baloncesto, este estudio buscó verificar la relación entre la estabilidad del core y el equilibrio postural en el rendimiento atlético en practicantes de baloncesto adolescentes, y si hay algún efecto positivo de estos entrenamientos específicos. Mediante la revisión de artículos que abordan la relación de estos factores en jóvenes deportistas de baloncesto, se investiga si existe una influencia significativa con un análisis de datos cualitativos.

\section{Metodología}

La planificación y estructuración de esteestudio se basaron en el «PRISMA statement»para producir una revisión sistemática, elas preguntas de investigación fueron referenciadas según la estrategia PICO (Population, Intervention, Comparison y Outcome) como se muestra en la tabla 1 (Moher et al., 2009).

La búsqueda se realizó en las bases de datos PubMed y Scielo en idioma inglés. Se utilizaron descriptores indexados en MeSH (Medical Subject Headings). Sin embargo, era necesario incluir descriptores de vocabulario no controlados*.

\begin{tabular}{|c|c|c|c|}
\hline Esquema de busqueda & & & \\
\hline $\begin{array}{l}\text { Términos de búsqueda } \\
\text { adolescents OR young } \\
\text { athletes OR/AND } \\
\text { basketball }\end{array}$ & $\begin{array}{l}\text { PICOS } \\
\text { Population }\end{array}$ & $\begin{array}{l}\text { Criterios de inclusión } \\
\text { Jóvenes deportistas de } \\
\text { baloncesto (hasta } 23 \text { años) }\end{array}$ & $\begin{array}{l}\text { Criterios de exclusión } \\
\text { Sin jugadores de baloncesto } \\
\text { con edad establecida }\end{array}$ \\
\hline $\begin{array}{l}\text { postural balance OR } \\
\text { balance OR balance } \\
\text { training* OR abdominal }\end{array}$ & Intervention & $\begin{array}{l}\text { Presencia de entrenamiento } \\
\text { de EC y / o EP }\end{array}$ & $\begin{array}{l}\text { Sin entrenamiento de } \mathrm{EC} y \\
\text { / o EP }\end{array}$ \\
\hline $\begin{array}{l}\text { muscles OR core } \\
\text { stability* OR core* OR } \\
\text { core training* }\end{array}$ & Comparision & $\begin{array}{l}\text { Comparación con el grupo } \\
\text { de control u otro } \\
\text { entrenamiento }\end{array}$ & Sin grupo de control \\
\hline $\begin{array}{l}\text { athletic performance OR } \\
\text { muscle strength }\end{array}$ & Outcome & $\begin{array}{l}\text { Prueba de rendimiento } \\
\text { atlético, como salto } \\
\text { vertical, cambio de } \\
\text { dirección, etc }\end{array}$ & $\begin{array}{l}\text { Sin prueba de rendimiento } \\
\text { atlético }\end{array}$ \\
\hline
\end{tabular}

Se identificaron y excluyeron estudios duplicados. Los demás pasaron por el proceso de lectura de títulos y resúmenes, se seleccionaron los estudios que cumplían con los criterios antes mencionados o generaban dudas para su posterior análisis del texto completo. Los trabajos que cumplieron con las especificaciones luego de estos pasos fueron incluidos en esta revisión. Este proceso fue realizado por un investigador que manejó los 
registros bibliográficos a través del software EndNote Online y Microsoft Excel, con el fin de facilitar la organización, búsquedas y análisis de los datos.

Una vez seleccionados los artículos, la extracción de datos se realizó considerando principalmente puntos como edad y sexo, diseño (tiempo y frecuencia de realización), pruebas aplicadas, tipo de entrenamiento prescrito, resultados observados y posibles explicaciones y consideraciones sobre los resultados del estudio. Con la relectura de cada artículo seleccionado, se tomaron notas específicas sobre cada uno de los estudios considerando los puntos mencionados para facilitar la identificación de estos datos y para la posterior síntesis cualitativa.

\section{Resultados}

La Figura 1 representa la dinámica de identificación, selección, elegibilidad, inclusión y número de estudios en cada etapa.

Las búsquedas en las bases de datos presentaron 282 artículos. Se identificaron y excluyeron 99 estudios duplicados.En la selección, se excluyeron 172 artículos, de los cuales 154 se descartaron de inmediato por no ajustarse al tema o se identificaron como artículos de
revisión.Los 18 trabajos restantes se examinaron más a fondo y se descartaron 13 por utilizar una población diferente, no tener pruebas de rendimiento deportivo o por fallas metodológicas. Los 5 trabajos que cumplieron con las especificaciones luego de estos pasos fueron incluidos en esta revisión.

La Tabla 2 presenta datos descriptivos sobre las características de las muestras de estos estudios. La suma de las muestras ascendió a 153 participantes, siendo el 130 mujeres y el 23 hombres. La edad media de la muestra total fue de 17,6 años.

En todos los protocolos hay entrenamiento de EC y / o EP, y se pueden destacar los siguientes tres tipos: equilibrio sobre plataforma inestable y fortalecimiento del core. Se realizaron solos o dentro de un programa combinado, junto con otros métodos de entrenamiento como ejercicios pliométricos, de resistencia, velocidad y agilidad. Todos los programas se llevaron a cabo con nuevos participantes. Es decir, al inicio de una sesión con otros componentes (inmediatamente después del calentamiento o como calentamiento) o de forma aislada de otras sesiones de entrenamiento.

El entrenamiento del equilibrio está marcado por la presencia de inestabilidad, ya sea con el uso de disposi-

Tabla 2

Características de los estudios seleccionados

\begin{tabular}{|c|c|c|c|c|c|c|c|}
\hline \multicolumn{2}{|l|}{ Autores / Año de } & \multirow{2}{*}{$\begin{array}{l}\text { Muestra } \\
\text { Edad (media / DE) }\end{array}$} & \multicolumn{2}{|c|}{ Diseño del estudio } & \multirow{3}{*}{ Frecuencia } & \multirow[b]{2}{*}{ Características del entrenamiento } & \multirow[b]{2}{*}{ Pruebas de desempeño atlético } \\
\hline Publicación & Tamaño & & grupos & Semanas & & & \\
\hline & & & & & & (Intervención) & (pre y post intervención) \\
\hline $\begin{array}{l}\text { Boccolini y col. } \\
\text { (2013) }\end{array}$ & 23 chicos & $15 \pm 014,6 \pm 0,1$ & $\begin{array}{l}\text { BAL (entrenamiento de } \\
\text { equilibrio) e ISO } \\
\text { (entrenamiento de } \\
\text { fuerza tradicional) }\end{array}$ & 12 & 2 & $\begin{array}{l}\text { Equilibrio estático y equilibrio dinámico } \\
\text { con tarea, ambos sobre una superficie } \\
\text { inestable. ISO realizó entrenamiento en } \\
\text { prensa de piernas y silla de extensión. } \\
\text { Ambos grupos recibieron entrenamiento } \\
\text { regular. }\end{array}$ & $\begin{array}{l}\text { BAL mostró una mejora significativa en el equilibrio sostenido } \\
\text { (bipodalic } 28.3 \% \text {; derecha } 41.4 \% \text {; izquierda } 45.8 \% \text { ) e en salto con } \\
\text { contramovimiento (CMJ) (bipodalic } 8.1 \% \text {; derecha } 13.5 \% \text {; } \\
\text { izquierda } 12.5 \% \text { ). El grupo ISO mejorou en equilibrio } \\
\text { monopodálico izquierdo (izquierda: } 16,39 \% \text { ). }\end{array}$ \\
\hline $\begin{array}{l}\text { Bouteraa y col. } \\
\text { (2020) }\end{array}$ & 26 chicas & $16,4 \pm 0,5 \quad 16,5 \pm 0,5$ & Experimental y Control & 8 & 2 & $\begin{array}{l}\text { Tres ejercicios de equilibrio sobre una } \\
\text { superficie inestable (estático y dinámico } \\
\text { con tarea) y tres ejercicios pliométricos } \\
\text { (horizontal y vertical). El grupo de } \\
\text { control realizó solo entrenamiento } \\
\text { regular. }\end{array}$ & 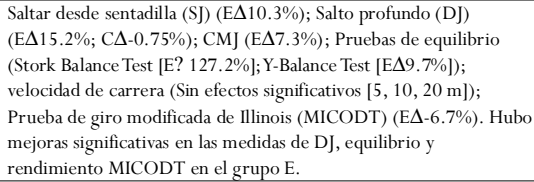 \\
\hline $\begin{array}{l}\text { Myer y col. } \\
\text { (2005) }\end{array}$ & 53 chicas & $15,3 \pm 0,916,5 \pm 1$ & $\begin{array}{l}\text { Grupo de } \\
\text { entrenamiento y grupo } \\
\text { de control }\end{array}$ & 6 & 3 & $\begin{array}{l}\text { El entrenamiento neuromuscular } \\
\text { dinámico incluyó pliometría, } \\
\text { fortalecimiento del core, entrenamiento } \\
\text { de equilibrio, entrenamiento de } \\
\text { resistencia y entrenamiento de velocidad } \\
\text { por intervalos. }\end{array}$ & $\begin{array}{l}\text { Altura de salto vertical }(39.9 \pm 0.9 \mathrm{~cm} \text { to } 43.2 \pm \\
1.1 \mathrm{~cm}) ; \text { Prueba de velocidad } 9.1 \mathrm{~m}(1.80 \pm 0.02 \text { seconds to } 1.73 \\
\pm 0.01 \text { seconds); Prueba de salto de longitud unilateral } \\
\text { manteniendo la posición en el aterrizaje (right, } 165.1 \pm 3.0 \mathrm{~cm} \text { to } \\
175.5 \pm 2.6 \mathrm{~cm} \text {; left, } 165.1 \pm 2.7 \mathrm{~cm} \text { to } 173.6 \pm 2.5 \mathrm{~cm}) ; \text { Prueba } \\
\text { de fuerza (sentadillas }[34.2 \pm 1.1 \mathrm{~kg} \text { to } 65.7 \pm 1.9 \mathrm{~kg}] \text { y press de } \\
\text { banca }[32.0 \pm 0.6 \mathrm{~kg} \text { to } 38.4 \pm 0.8 \mathrm{~kg}]) ; \text { Análisis biomecánico } \\
\text { tridimensional de DJ. }\end{array}$ \\
\hline $\begin{array}{l}\text { Sasaki y col. } \\
\text { (2019) }\end{array}$ & 17 mujeres & $19,7 \pm 0,920,3 \pm 2,5$ & $\begin{array}{l}\text { Grupo de } \\
\text { entrenamiento y grupo } \\
\text { de control }\end{array}$ & 8 & 4 o más & $\begin{array}{l}\text { El programa de entrenamiento básico } \\
\text { inclúa solo } 3 \text { componentes del programa } \\
\text { FIFA11 +: tabla isométrica, tabla } \\
\text { isométrica lateral y flexión nórdica. El } \\
\text { grupo de control realizó solo } \\
\text { entrenamiento regular. }\end{array}$ & $\begin{array}{l}\text { Sistema de análisis de movimiento tridimensional: evaluación } \\
\text { a cinemática y cinética de miembros inferiores y tronco durante DJ y } \\
\text { sentadilla; Medición de la fuerza de los músculos isocinéticos: } \\
\text { flexor y abductor de la cadera; flexor y extensor de rodilla y fuerza } \\
\text { extensora y flexora del tronco. Después del entrenamiento de los } \\
\text { músculos del core, el pico torsión de los flexores de rodilla a } 60^{\circ} / \mathrm{s} \\
\text { y } 120^{\circ} / \mathrm{s} \text { y los flexores del tronco a } 20^{\circ} / \mathrm{s} \text { fue mayor en el grupo de } \\
\text { entrenamiento. }\end{array}$ \\
\hline $\begin{array}{l}\text { Zemková y } \\
\text { Hamar (2010) }\end{array}$ & 34 mujeres & $20,9 \pm 2,421,2 \pm 2,8$ & Experimental y Control & 6 & 4 a 5 & $\begin{array}{l}\text { El entrenamiento combinó agilidad y } \\
\text { equilibrio. Tareas dinámicas (por } \\
\text { ejemplo, atrapar la pelota) manteniendo } \\
\text { simultáneamente el equilibrio sobre una } \\
\text { superficie inestable . El grupo de control } \\
\text { realizó el mismo protocolo en una } \\
\text { superficie estable. }\end{array}$ & 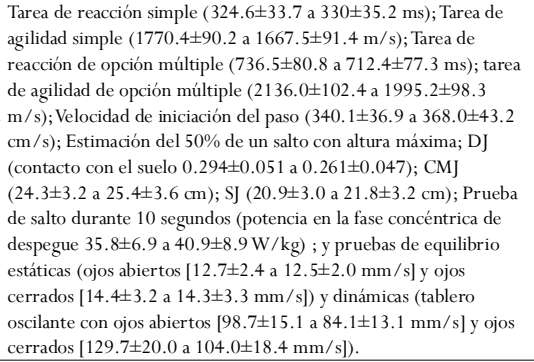 \\
\hline
\end{tabular}


tivos desestabilizadores (disco de equilibrio, pelota suiza, BOSU, etc.) o con desestabilización constante (ejercicios de resistencia, lanzamiento o recepción de pelota, uso de bandas elásticas, etc.), o con ambos (Zech et al., 2010). La musculatura central se puede entrenar de diferentes formas. Los ejercicios aislados, con acciones isométricas, largos períodos de tensión y menos carga tienden a contribuir a la fuerza del core.

El componente de inestabilidad (entrenamiento de equilibrio) también es un medio para aumentar la actividad del core (disco de equilibrio, pelota suiza, BOSU, etc.). Los ejercicios de resistencia tradicionales con peso libre generan una activación moderada del core, suficiente para desarrollar la estabilidad y mantener una postura neutra.Y aún pueden modificarse para enfatizar el core (Willardson, 2007; Hibbs et al., 2008; Wirth et al., 2017).

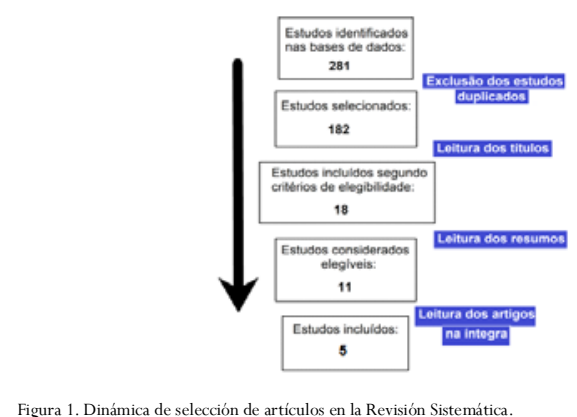

El tiempo de intervención varió de 6 a 12 semanas y la frecuencia semanal de entrenamiento varió de dos a cinco veces por semana. En cuanto la intervención, 2estudios presentaron solo programas específicos de EC o EP y 3 presentaron programas con estos entrenamientos combinados con otros tipos.

En la Tabla 3 se presentan las principales considera-

\begin{tabular}{|c|c|}
\hline Publicación & Resumen / consideraciones de resultados \\
\hline $\begin{array}{l}\text { Boccolini y } \\
\text { col. (2013) }\end{array}$ & $\begin{array}{l}\text { El estudio sugiere que el entrenamiento del equilibrio con tablas inestables es un } \\
\text { método eficaz para mejorar el equilibrio y el salto vertical, especialmente para los } \\
\text { jóvenes atletas aficionados. Este es un método de entrenamiento asequible que } \\
\text { todo entrenador de baloncesto debería considerar. }\end{array}$ \\
\hline $\begin{array}{l}\text { Bouteraa y } \\
\text { col. (2020) }\end{array}$ & $\begin{array}{l}\text { Las principales conclusiones fueron de más mejoras en el grupo Experimental que } \\
\text { en el grupo Control con respecto al rendimiento de DJ, balance y MICODT. Sin } \\
\text { embargo, el programa combinado no logró ganancias significativamente mayores } \\
\text { en SJ, CMJ o velocidad de carrera. El estudio indica que las jugadoras de } \\
\text { baloncesto se benefician de una combinación de equilibrio y pliometría en un } \\
\text { programa de entrenamiento durante la temporada. }\end{array}$ \\
\hline $\begin{array}{l}\text { Myer y col. } \\
\text { (2005) }\end{array}$ & $\begin{array}{l}\text { El estudio concluye que un programa de entrenamiento neuromuscular combinado } \\
\text { diseñado para la prevención de lesiones en las extremidades inferiores puede } \\
\text { proporcionar mejoras simultáneas en el rendimiento deportivo y la biomecánica } \\
\text { del movimiento en atletas femeninas. Y sugiere que los programas de } \\
\text { acondicionamiento de pretemporada incluyen componentes de pliometrí, } \\
\text { entrenamiento de resistencia, fortalecimiento del núcleo, entrenamiento de } \\
\text { equilibrio y velocidad. }\end{array}$ \\
\hline $\begin{array}{l}\text { Sasaki y col. } \\
(2019)\end{array}$ & $\begin{array}{l}\text { El estudio sugiere que realizar un entrenamiento básico simple resultó en cambios } \\
\text { en el control neuromuscular en el tronco y las extremidades inferiores en los } \\
\text { jugadores de baloncesto, lo que podría reducir el riesgo de lesión del ligamento } \\
\text { cruzado anterior sin contacto. }\end{array}$ \\
\hline $\begin{array}{l}\text { Zemková y } \\
\text { Hamar } \\
\text { (2010) }\end{array}$ & $\begin{array}{l}\text { El entrenamiento combinado de agilidad y equilibrio mejoró el equilibrio } \\
\text { dinámico, aumentó la velocidad de paso inicial, mejoró el rendimiento de la } \\
\text { agilidad y redujo el tiempo de contacto con el suelo en el salto profundo. Sin } \\
\text { embargo, considera que el entrenamiento es insuficiente para mejorar el tiempo } \\
\text { de reacción de simple y múltiple opción y la potencia de los miembros inferiores. } \\
\text { Sugiere que los ejercicios de equilibrio realizados con tareas de reacción pueden } \\
\text { considerarse un medio eficaz para mejorar el rendimiento neuromuscular en } \\
\text { deportistas de élite. }\end{array}$ \\
\hline
\end{tabular}

ciones de cada estudio, con el efecto del entrenamiento aplicado sobre las diferentes variables de rendimiento deportivo analizadas, a partir de la traducción y algunas modificaciones de los extractos originales de los estudios.

Los dos estudios que propusieron un programa de entrenamiento combinado (Bouteraa y col., 2020; Sasaki y col., 2019) mostraron resultados de mejora significativa del rendimiento. Los estúdios de Sasaki y col. (2019), y Zemková y Hamar (2010) utilizaron protocolos específicos de EC o EP, obteniendo resultados positivos en aspectos como la fuerza isométrica máxima de la musculatura de los miembros inferiores y la mejora de los patrones de movimiento, por lo que se consideran medios efectivos para mejorar el control neuromuscular, pero sin efectos resultados directos significativos en el rendimiento atlético.

El estudio de Boccolini y col. (2013) propuso un entrenamiento adicional de EP, sin embargo se utilizaron ejercicios dinámicos sobre una superficie inestablecon una progresión a lo largo del estudio, obteniendo mejoras significativas en el rendimiento deportivo.

Entre las variables de rendimiento deportivo con efectos significativos, destaca el salto vertical (Bouteraa y col., 2020; Boccolini y col., 2013; Myer y col., 2005), pero también aparecen mejoras en las pruebas de cambio de dirección (Bouteraa y col., 2020) y agilidad (Zemková y Hamar, 2010), como se muestra en la tabla 2.

$\mathrm{Al}$ analizar esta información sobre el entrenamiento utilizado en relación al tiempo de intervención propuesto en los estudios, de 6 a 8 semanas fueron suficientes para percibir el desarrollo del rendimiento en los estudios con entrenamiento combinado (Bouteraa y col., 2020; Myer y col., 2005). Em el trabajos con solo programas específicos de EC o EP, aquello que mostraron mejoras significativas en el rendimiento deportivo (Boccolini y col., 2013) aplicaron el entrenamiento por un período más largo (12 semanas), mientras que el otro (Zemková y Hamar, 2010) tuvo una intervención de 6 semanas y no mostró mejora en el rendimiento deportivo.

\section{Discusión}

Entre los cinco estudios seleccionados, tres mostraron algun efecto positivo sobre el rendimiento deportivo de los jóvenes atletas de baloncesto,entre estos solo uno utilizó una intervención adicional específica para EC y / o EP, sin ningún otro tipo de entrenamiento en 
conjunto. Estos datos aislados corroboran los hallazgos en la literatura, señalando que no hay evidencia sólida de la efectividad de estos entrenamientos en rendimiento deportivo (Wirth et al., 2017; Cressey et al., 2007).

Chaouachi et al. (2014) y Distefano et al. (2013), quienes presentaron programas de entrenamiento con tales características (ej. Equilibrio dinámico, saltos, etc.) en deportistas jóvenes y observaron efectos positivos en algunos aspectos como el rendimiento desprints, cambio de dirección y saltos. Por el contrario, Prieske et al. (2016) utilizaron un programa compuesto esencialmente por ejercicios isométricos de EC / EP y no observaron resultados significativos en estos aspectos en jóvenes deportistas de fútbol.

Granacher y Gollhofer (2011) sugieren que existe una asociación positiva entre la fuerza isométrica y dinámica en los adolescentes, lo que implica que el aumento de la fuerza isométrica después del entrenamiento de resistencia puede transferirse en cierta medida al rendimiento del salto vertical, por ejemplo.Los resultados de los protocolos isométricos se han utilizado como indicadores de la fuerza atlética, la maximal voluntary contraction (MVC) se puede desarrollar sin un cambio en el ángulo de la articulación contra un objeto inamovible, medido en Newtons (N). La tasa de desarrollo de la fuerza (TDF) es una medida de la potencia atlética que considera el primer período (60 a $100 \mathrm{~ms}$ ) de la MVC.A razón para el uso de TDF es que en muchos deportes el tiempo disponible para generar fuerza es limitado. Sin embargo, existe una discusión de que las pruebas isométricas guardan poca semejanza con la naturaleza dinámica de la mayoría de las tareas deportivas (Abernethy, Wilson y Logan, 1995). Es de destacar que el nivel de maduración del individuo influye en esta variable, y luego del pico de velocidad de crecimiento (PVC) hay mayor desarrollo de fuerza y potencia, y en las mujeres este pico ocurre antes (McQuilliam y col., 2020).

Colson et al. (2010) mostró un aumento significativo en la fuerza isométrica máxima de los músculos extensores de la rodilla después del entrenamiento en una plataforma vibratoria, sin embargo, el estudio no encontró una transferencia a la fuerza dinámica. Por tanto, los autores sugieren que el entrenamiento del equilibrio debe realizarse como complemento a otros métodos de entrenamiento,con el propósito de prevenir lesiones (Colson et al., 2010; Granacher \& Gollhofer, 2011).

Así, los hallazgos de esta revisión sistemática se refieren a la comprensión del entrenamiento de EC / EP como una forma de desarrollar el control neuromuscularde los practicantes de baloncesto adolescentes. Sin embargo, todavía no está claro si esto puede tener alguna influencia en los indicadores de rendimiento deportivo y conducir a mejores resultados. Así, este estudio trae reflexiones que solo permiten la formulación de hipótesis sobre el tema, dadas sus limitaciones.

Entre las limitaciones, se puede señalar la escasez de estudios con la población adolescente y especialmente con los practicantes de baloncesto adolescentes, además de que la temática presenta conceptos que aún no están del todo establecidos en cuanto a términos y definiciones, dando lugar a una amplia variedad de protocolos de entrenamiento encontrados.

Finalmente, el análisis de esta revisión de la literatura no contempló ni enfatizó algunos puntos debido al número limitado de artículos, tales como: adaptaciones fisiológicas al entrenamiento; el período de la temporada que se celebró; la duración de la sesión; y la relación con las habilidades técnicas deportivas de la modalidad. En este sentido, se necesitan futuras investigaciones sobre el tema para comprender mejor la relación con el rendimiento deportivo y definir protocolos de entrenamiento más adecuados, con el objetivo de verificar las adaptaciones provocadas en niños y adolescentes. Así como intentar establecer una frecuencia y volumen suficientes para incrementar el control neuromuscular, con el fin de ajustar con otras variables y combinaciones con otros métodos.Como se desprende del reducido número de trabajos incluidos, es un tema poco investigado pero con mucho uso práctico.

\section{Conclusión}

De acuerdo con los resultados obtenidos, es posible observar que el efecto significativo sobre el rendimiento deportivo verificado en algunos estudios puede estar relacionado con el nivel de entrenamiento y la edad de los participantes, así como con la característica dinámica del entrenamiento aplicado. Sin embargo, en general, no fue posible identificar pruebas sólidas de la influencia positiva del entrenamiento de EC / EP en las habilidades biomotoras de los adolescentes practicantes de baloncesto.

\section{Aplicaciones práticas}

Este tipo de entrenamiento puede ser una alternativa interesante para entrenadores y preparadores físicos para complementar la formación de jóvenes deportis- 
tas de baloncesto e incluso en la iniciación deportiva, pensando en la mejora del control neuromuscular. También considerando que los entrenamientos son accesibles y seguros de aplicar, se pueden utilizar como calentamiento para la sesión y pensando en salvar a los deportistas del impacto que el propio juego y entrenamiento de baloncesto impone a altos niveles.

\section{Agradecimientos}

Los autores no tienen financiación ni conflictos de intereses que revelar. No se recibió financiación de ninguna organización.

\section{Referencias}

Abernethy, P., Wilson, G., \& Logan, P. (1995). Strength and power assessment. Sports medicine, 19(6), 401-417.

Albaladejo, M., Cristobal, R.V., \& Ros, F. E. (2019). Efecto del entrenamiento en pretemporada en las variables antropométricas y derivadas en jugadores de baloncesto de élite. Retos: nuevas tendencias en educación física, deporte y recreación, (36), 474-479.

Behm, DG, Drinkwater, EJ, Willardson, JM y Cowley, PM (2010). Posición de posición de la Sociedad Canadiense de Fisiología del Ejercicio: El uso de la inestabilidad para entrenar el núcleo en acondicionamiento atlético y no atlético. Fisiología aplicada, nutrición y metabolismo, 35 (1), 109-112.

Boccolini, G., Brazzit, A., Bonfanti, L. y Alberti, G. (2013). Utilizar el entrenamiento del equilibrio para mejorar el rendimiento de los jugadores de baloncesto juveniles. Ciencias del deporte para la salud, 9 (2), 37-42.

Bompa, T. O. (2002). Periodização e metodologia do treinamento. $1^{a}$ Edição. São Paulo, SP,Phorte Editora.

Bouteraa, I., Negra, Y., Shephard, RJ y Chelly, MS (2020). Efectos del equilibrio combinado y el entrenamiento pliométrico sobre el rendimiento deportivo en jugadoras de baloncesto. The Journal of Strength \& Conditioning Research, 34 (7), 1967-1973.

Chaouachi, A., Othman, AB, Hammami, R., Drinkwater, EJy Behm, DG (2014). La combinación de entrenamiento pliométrico y de equilibrio mejora el rendimiento de las carreras de velocidad y lanzadera con más frecuencia que el entrenamiento solo pliométrico con niños. The Journal of Strength \& Conditioning Research, 28 (2), 401-412.

Chappell, JD y Limpisvasti, O. (2008). Efecto de un programa de entrenamiento neuromuscular sobre la cinética y cinemática de las tareas de salto. The American Journal of Sports Medicine, 36 (6), 1081-1086.

Colson, SS, Pensini, M., Espinosa, J., Garndes, F. y Legros, P.
(2010). Efectos del entrenamiento con vibraciones de cuerpo entero sobre el rendimiento físico de los jugadores de baloncesto.The Journal of Strength \& Conditioning Research, 24 (4), 999-1006.

Cressey, EM,West, CA,Tiberio, DP, Kraemer,WJ y Maresh, CM(2007). Los efectos de diez semanas de entrenamiento de superficie inestable de la parte inferior del cuerpo sobre los marcadores de rendimiento atlético. Revista de investigación de fuerza y acondicionamiento, 21 (2), 561.

DiStefano, LJ, DiStefano, MJ, Frank, BS, Clark, MA y Padua, DA (2013). Comparación de entrenamiento integrado y aislado sobre medidas de rendimiento y control neuromuscular. The Journal of Strength \& Conditioning Research, 27 (4), 1083-1090.

Fachina, R., da Silva, A., Falcão, W., Montagner, P., Borin, J., Minozzo, F., ... y de Lira, C. (2013). La influencia de la vibración de todo el cuerpo en la actividad de la creatina quinasa y el rendimiento de salto en jóvenes jugadores de baloncesto. Investigación trimestral para ejercicio y deporte, 84(4), 503-511.

Fort,A., Romero, D., Bagur, C. y Guerra, M. (2012). Efectos del entrenamiento con vibraciones de cuerpo entero sobre la fuerza explosiva y el control postural en atletas jóvenes. The Journal of Strength \& Conditioning Research, 26 (4), 926-936.

Gollhofer, A. (2003). Proprioceptive training: considerations for strength and power production. Strength and power in sport, 2, 331-342.

Granacher, U. y Gollhofer,A. (2011). ¿Existe asociación entre variables de control postural y fuerza en adolescentes ?.The Journal of Strength \& Conditioning Research, 25 (6), 1718-1725.

Granacher, U. y Gollhofer, A. (2012). ¿Existe asociación entre variables de control postural y fuerza en niños prepúberes ?. The Journal of Strength \& Conditioning Research, 26(1), 210-216.

Granacher, U., Muehlbauer,T., Maestrini, L., Zahner, L. y Gollhofer,A. (2011). ¿Puede el entrenamiento del equilibrio promover el equilibrio y la fuerza en niños prepúberes? The Journal of Strength \& Conditioning Research, 25 (6), 1759-1766.

Hammami, R., Chaouachi,A., Makhlouf, I., Granacher, U. y Behm, DG (2016). Asociaciones entre equilibrio y fuerza muscular, rendimiento de potencia en atletas jóvenes masculinos de diferente estado de madurez. Ciencias del ejercicio pediátrico, 28 (4), 521-534.

Hibbs, A. E., Thompson, K. G., French, D., Wrigley, A., \& Spears, I. (2008). Optimizing performance by improving core stability and core strength. Sports medicine, 38(12), 995-1008.

Hoshikawa,Y., Iida,T., Muramatsu, M., Ii, N., Nakajima,Y., Chumank, K. y Kanehisa, H. (2013). Efectos del entrena- 
miento de estabilización sobre la musculatura del tronco y el rendimiento físico en futbolistas juveniles. The Journal of Strength \& Conditioning Research, 27 (11), 3142 3149.

Hrysomalis, C. (2011). Equilibrar la capacidad y el rendimiento deportivo. Medicina deportiva, 41 (3), 221-232.

Junior, A .; Gomes, AC. Deporte: Preparación de jóvenes deportistas ( $\left.1^{\mathrm{a}} \mathrm{ed}\right)$. Londrina: Editorial de entrenamiento deportivo, 2014.

Kibler,W. B., Press, J., \& Sciascia, A. (2006).The role of core stability in athletic function. Sports medicine, 36(3), 189. 198.

McInnes, SE, Carlson, JS, Jones, CJ y McKenna, MJ (1995). La carga fisiológica impuesta a los jugadores de baloncesto durante la competición. Revista de ciencias del deporte, 13 (5), 387-397.

McQuilliam, S. J., Clark, D. R., Erskine, R. M., \& Brownlee, T. E. (2020). Free-weight resistance training in youth athletes: A narrative review. Sports Medicine, 50(9), 1567 1580 .

Moher, D. , Liberati, A., Tetzlaff, J., Altman, DG y Prisma Group. (2009). Elementos de informe preferidos para revisiones sistemáticas y metanálisis: la declaración PRISMA. Medicina PLoS, 6 (7), e1000097.

Myer, GD, Ford, KR, PALUMBO, OP y Hewett, TE (2005). El entrenamiento neuromuscular mejora el rendimiento y la biomecánica de las extremidades inferiores en atletas femeninas. The Journal of Strength \& Conditioning Research, 19(1), 51-60.

Narazaki, K., Berg, K., Stergiou, N., \& Chen, B. (2009). Physiological demands of competitive basketball. Scandinavian journal of medicine \& science in sports, 19(3), 425-432.

Okada,T., Huxel, KC y Nesser,TW (2011). Relación entre estabilidad central, movimiento funcional y rendimiento. The Journal of Strength \& Conditioning Research, 25 (1), 252-261.

Plisky, PJ, Rauh, MJ, Kaminski,TWy Underwood, FB (2006). Prueba de equilibrio Star Excursion como predictor de lesiones en las extremidades inferiores en jugadores de baloncesto de la escuela secundaria. Revista de fisioterapia ortopédica y deportiva, 36 (12), 911-919.

Prieske, O. , Muehlbauer,T.y Granacher, U. (2016). El papel de la fuerza de los músculos del tronco para la aptitud física y el rendimiento atlético en individuos entrenados: una revisión sistemática y un metanálisis. Medicina deportiva, 46 (3), 401-419.

Prieske, O., Muehlbauer,T., Borde, RA, Gube, M., Bruhn, S., Behm, DG y Granacher, U. (2016). Rendimiento neuromuscular y atlético después del entrenamiento de fuerza central en el fútbol juvenil de élite: papel de la inestabilidad. Revista escandinava de medicina y ciencia en el deporte, 26 (1), 48-56.

Richardson, CA, Snijders, CJ, Hides, JA, Damen, L., Pas, MS y Storm, J. (2002). La relación entre los músculos transverso del abdomen, la mecánica de la articulación sacroilíaca y el dolor lumbar. Columna vertebral, 27 (4), 399-405.

Santos, EJ y Janeira, MA (2008). Efectos del entrenamiento complejo sobre la fuerza explosiva en jugadores de baloncesto varones adolescentes. The Journal of Strength \& Conditioning Research, 22 (3), 903-909.

Santos, EJ y Janeira, MA (2012). Los efectos del entrenamiento de resistencia sobre los indicadores de fuerza explosiva en jugadores de baloncesto adolescentes. The Journal of Strength \& Conditioning Research, 26 (10), 2641-2647.

Sasaki, S.,Tsuda, E.,Yamamoto,Y., Maeda, S., Kimura,Y., Fujita, Y. e Ishibashi,Y. (2019). Entrenamiento core-muscular y control neuromuscular de miembros inferiores y tronco. Revista de entrenamiento atlético, 54(9), 959-969.

Schelling, X., \& Torres-Ronda, L. (2013). Conditioning for basketball: Quality and quantity of training. Strength \& Conditioning Journal, 35(6), 89-94.

Sharrock, C., Cropper, J., Mostad, J., Johnson, M. y Malone, T. (2011). Un estudio piloto de estabilidad del core y rendimiento deportivo: ¿existe una relación?. Revista internacional de fisioterapia deportiva, $6(2), 63$.

Shinkle, J., Nesser, TW, Demchak, TJ y McMannus, DM (2012). Efecto de la fuerza del núcleo sobre la medida de la potencia en las extremidades. The Journal of Strength \& Conditioning Research, 26 (2), 373-380.

Willardson, JM (2007). Entrenamiento de estabilidad del core: aplicaciones a programas de acondicionamiento deportivo. The Journal of Strength \& Conditioning Research, 21 (3), 979-985.

Wirth, K., Hartmann, H., Mickel, C., Szilvas, E., Keiner, M.y Sander,A. (2017). Estabilidad del core en deportistas: un análisis crítico de las directrices actuales. Medicina deportiva, 47(3), 401-414.

Zech,A., Hübscher, M.,Vogt, L., Banzer,W., Hänsel, F. y Pfeifer, K. (2010). Entrenamiento del equilibrio para el control neuromuscular y la mejora del rendimiento: una revisión sistemática. Revista de entrenamiento atlético, 45 (4), $392-$ 403.

Zemková, E. y Hamar, D. (2010). El efecto del entrenamiento combinado de agilidad-equilibrio de 6 semanas sobre el rendimiento neuromuscular en jugadores de baloncesto. Revista de medicina deportiva y aptitud física, 50 (3), 262267. 